\title{
Proton Pump Inhibitors and Osteoporosis: Is Collagen a Direct Target?
}

\author{
Yohannes T. Ghebre ${ }^{1,2 *}$ \\ ${ }^{1}$ Department of Radiation Oncology, Baylor College of Medicine, Houston, TX, United States, ${ }^{2}$ Department of Medicine, \\ Section on Pulmonary and Critical Care Medicine, Baylor College of Medicine, Houston, TX, United States
}

Keywords: proton pump inhibitors, osteoporosis, osteoporotic fractures, bone density, collagen

\section{BACKGROUND}

Proton pump inhibitors (PPIs) are FDA-approved drugs for the treatment of several gastric acid-related disorders including indigestion, peptic ulcers, Barrett's esophagus, Zollinger Ellison syndrome and Helicobacter pylori infection-induced gastritis (1). Accordingly, PPIs are among the most consumed drugs worldwide with an ever-increasing use from time to time (2). Currently, the global sales of prescription and over-the-counter PPIs is estimated to be about $\$ 14$ billion dollars with $\sim 113$ million PPI prescriptions worldwide (3). One of the growing concerns with widespread PPI use is their inappropriate use for prolonged period of time to treat gastric acidity, as well as for indications that are not quite clear. By extension, one of the most significant medical concerns is the potential association of PPI use with increased risk for osteoporotic fractures including hip and vertebral fractures (4-6). Recently, a large meta-analysis study reported that the use of PPIs is significantly associated with increased risk of hip fracture (7). Accordingly, the US FDA has issued a safety warning label in 2010 to emphasize the increased risk of spine, wrist and hip fractures with PPI use. These concerns for musculoskeletal harm are almost exclusively believed to be due to reduced absorption of calcium presumably because PPIs cause low level of stomach acid (hypochlorhydria) that is believed to impair calcium solubility and cause malabsorption to exacerbate loss of bone mineral density secondary to low plasma calcium-induced hyperparathyroidism, osteoclast activation and bone resorption $(8,9)$. Intriguingly, there appears to be a trend toward increased bone fracture incidence with increased dose, adherence and duration of PPI use, but not the use of histamine $\mathrm{H}_{2}$-receptor antagonists (H2RAs; alternate antacids with similar acid-reducing capabilities as PPIs) (10), and that this risk is independent of changes in bone mineral density $(4,8,11,12)$ suggesting that the depletion of bone minerals such as calcium and vitamin $\mathrm{D}$ or suppression of gastric acidity is unlikely to be the driving mechanism by which PPIs increase the risk of osteoporotic fractures. Additional clinical studies have observed that PPI users are more likely to sustain fracture and start medications for osteoporosis than non-users (13), and that there was no fracture risk reduction with the use of hormone replacement therapy (HRT) if PPIs are concomitantly used (14) indicating the increased risk of fracture with PPI use and possible negation of the bone protective effect of HRT. Alternative explanations for the increased risk of osteoporotic fracture with PPI use include inhibition of osteoblast or osteoclast activity to dysregulate bone remodeling $(15,16)$, and increased rate of bone resorption (17). However, clinical cohorts comparing long term PPI users with non-users found no difference in the rate of bone density loss including in perimenopause women who are at increased risk of bone loss and potentially susceptible to an adverse effect $(18,19)$. Intriguingly, Jo et al. $(17)$ reported that the use of PPI was associated with increased release of calcium and deoxypyridinoline; a crosslink that provides structural stiffness to the type I collagen found in bones, suggesting that major components of the bone might be targeted by PPIs. 


\section{PERSPECTIVE}

Recently, our cell biological and preclinical animal studies revealed that PPIs target major extracellular matrix (ECM) components such as collagen (20). Our in vitro studies in primary cells derived from human lungs indicate that PPIs inhibit the gene expression of collagen (e.g., collagen 1A1) and fibronectin, and PPI-treated lung fibroblasts show significantly reduced degree of proliferation and decreased collagen release (20). Our in vivo studies in an animal models exposed to chemotherapeutic drug (bleomycin) or radiation; stimuli known to induce fibrosis (ECM deposition), also showed that administration of the PPI esomeprazole significantly reduced collagen accumulation in the lungs and skin $(20,21)$. Other studies also reported that PPIs reduce collagen accumulation in the liver (22). Intriguingly, there is also a report that lung fibrosis patients who happened to be on PPIs had reduced fibrosis score on high resolution computed tomography (HRCT) scans (23) suggesting that PPIs might target collagen. Previously, Pullamsetti et al. (24) have reported that the expression of dimethylarginine dimethylaminohydrolase (DDAH), a cytoplasmic enzyme that is closely associated with inducible nitric oxide synthase (iNOS) to promote inflammatory and fibrotic phenotypes, is pathologically upregulated in the lungs of pulmonary fibrosis patients, and our biochemical and immunohistochemical studies $(20,21,25)$ show that PPIs inhibit DDAH to reduce soluble and total collagen levels. Our recent studies in human lung cells also show that PPIs inhibit the gene expression of collagen types 1, 3, and 5 . Accessible data from The Library of Integrative Network-based Cellular Signatures (LINCS) database (http://www.lincscloud. org) on lung cells treated with esomeprazole show that the PPI inhibits the expression of several ECM components including members of the collagen family such as collagen $3 \mathrm{~A} 1$ (COL3A1), collagen 4A1 (COL4A1), collagen 5A1 (COL5A1), collagen 5A2 (COL5A2), and other myofibroblast markers $(20,26)$. Taken together, it appears that PPIs may actually target the ECM in general and members of the collagen family in particular to influence bone pathophysiology including increasing the risk of osteoporosis and osteoporotic fractures in patients. In the face of lack of plausible biological mechanism and conclusive evidence for causal relationship between PPI use and osteoporosis, the new mechanistic insights provided here need to be investigated further. In addition, the lack of positive correlation between

\section{REFERENCES}

1. Strand DS, Kim D, Peura DA. 25 years of proton pump inhibitors: a comprehensive review. Gut Liver. (2017) 11:27-37. doi: 10.5009/gnl15502

2. Halfdanarson OO, Pottegard A, Bjornsson ES, Lund SH, Ogmundsdottir MH, Steingrimsson E, et al. Proton-pump inhibitors among adults: a nationwide drug-utilization study. Therap $A d v$ Gastroenterol. (2018) 11:1756284818777943. doi: 10.1177/17562848187 77943

3. Katz MH. Failing the acid test: benefits of proton pump inhibitors may not justify the risks for many users. Arch Intern Med. (2010) 170:7478. doi: 10.1001/archinternmed.2010.64 bone mineral density and osteoporotic fracture in PPI users as described above invites a fresh perspective into looking at these two conditions independent of each other. Prospective studies should also be designed to isolate bone cells and evaluate the gene expression of members of the collagen family that constitute the ECM including collagen types 1,3 , and 5 in susceptible patients prior to, during, and after the completion of PPI use. Such clinical studies may be preceded by preclinical assays of primary human osteoblasts (27) cultured in the absence or presence of PPIs, and analyzed for the expression of ECM proteins. It is anticipated that such prospective studies will address important questions including whether PPIs have direct effect on collagen and/or other major ECM components.

\section{CONCLUSION}

Since we cannot rule out the possibility of causal relationship between PPI use and osteoporosis, clinicians should be judicious with the prescription of PPIs and should vigilantly monitor at risk patients including older patients as well as long term PPI users.

\section{AUTHOR CONTRIBUTIONS}

The author confirms being the sole contributor of this work and has approved it for publication.

\section{FUNDING}

This study was supported (to YG) in part by grants from the NHLBI (grant numbers K01HL118683; R01HL137703), American Heart Association (grant number 17GRNT33460159), the Cancer Prevention and Research Institute of Texas (grant number RP190497), and by intramural funding from Baylor College of Medicine (project ID 2690000104).

\section{ACKNOWLEDGMENTS}

The author would like to thank the Departments of Radiation Oncology and Medicine at Baylor College of Medicine for overall support. The bioinformatics analysis is courtesy of Dr. Anil Jegga, Division of Biomedical Informatics, Cincinnati Children's Hospital Medical Center, Department of Pediatrics, University of Cincinnati College of Medicine, Cincinnati, Ohio. 
8. Nassar Y Richter S. Proton-pump inhibitor use and fracture risk: an updated systematic review and meta-analysis. J Bone Metab. (2018) 25:14151. doi: 10.11005/jbm.2018.25.3.141

9. Yang YX. Chronic proton pump inihibitor therapy and calcium metabolism. Curr Gastroenterol Rep. (2012) 14:473-9. doi: 10.1007/s11894-012-0290-4

10. Vestergaard P, Rejnmark L, Mosekilde L. Proton pump inhibitors, histamine $\mathrm{H} 2$ receptor antagonists, and other antacid medications and the risk of fracture. Calcif Tissue Int. (2006) 79:76-83. doi: 10.1007/s00223-006-0021-7

11. Yu EW, Bauer SR, Bain PA, Bauer DC. Proton pump inhibitors and risk of fractures: a meta-analysis of 11 international studies. Am J Med. (2011) 124:519-26. doi: 10.1016/j.amjmed.2011.01.007

12. Adams AL, Black MH, Zhang JL, Shi JM, Jacobsen SJ. Proton-pump inhibitor use and hip fractures in men: a population-based case-control study. Ann Epidemiol. (2014) 24:286-90. doi: 10.1016/j.annepidem.2014.01.004

13. van der Hoorn MMC, Tett SE, de Vries OJ, Dobson AJ, Peeters G. The effect of dose and type of proton pump inhibitor use on risk of fractures and osteoporosis treatment in older Australian women: a prospective cohort study. Bone. (2015) 81:675-82. doi: 10.1016/j.bone.2015.08.024

14. Moberg LM, Nilsson PM, Samsioe G, Borgfeldt C. Use of proton pump inhibitors (PPI) and history of earlier fracture are independent risk factors for fracture in postmenopausal women. The WHILA study. Maturitas. (2014) 78:310-5. doi: 10.1016/j.maturitas.2014.05.019

15. Prause M, Seeliger C, Unger M, Rosado Balmayor E, van Griensven M, Haug AT. Pantoprazole decreases cell viability and function of human osteoclasts in vitro. Mediators Inflamm. (2015) 2015:413097. doi: 10.1155/2015/413097

16. Costa-Rodrigues J, Reis S, Teixeira S, Lopes S, Fernandes MH. Dosedependent inhibitory effects of proton pump inhibitors on human osteoclastic and osteoblastic cell activity. FEBS J. (2013) 280:505264. doi: $10.1111 /$ febs. 12478

17. Jo Y, Park E, Ahn SB, Jo YK, Son B, Kim SH, et al. A proton pump inhibitor's effect on bone metabolism mediated by osteoclast action in old age: a prospective randomized study. Gut Liver. (2015) 9:60714. doi: $10.5009 /$ gnl14135

18. Targownik LE, Goertzen AL, Luo Y, Leslie WD. Long-term proton pump inhibitor use is not associated with changes in bone strength and structure. Am J Gastroenterol. (2017) 112:95-101. doi: 10.1038/ajg.2016.481

19. Solomon DH, Diem SJ, Ruppert K, Lian YJ, Liu CC, Wohlfart A, et al. Bone mineral density changes among women initiating proton pump inhibitors or H2 receptor antagonists: a SWAN cohort study. J Bone Miner Res. (2015) 30:232-9. doi: 10.1002/jbmr.2344

20. Ghebremariam YT, Cooke JP, Gerhart W, Griego C, Brower JB, DoyleEisele $\mathrm{M}$, et al. Pleiotropic effect of the proton pump inhibitor esomeprazole leading to suppression of lung inflammation and fibrosis. J Transl Med. (2015) 13:249. doi: 10.1186/s12967-015-0614-x

21. Pham N, Ludwig M, Wang M, Ebrahimpour A, Bonnen M, Diwan A, et al. Topical esomeprazole mitigates radiation-induced dermal inflammation and fibrosis. Radiation Res. (2019) 192:473-82. doi: 10.1667/RR15398.1

22. Eltahir HM Nazmy MH. Esomeprazole ameliorates $\mathrm{CCl} 4$ induced liver fibrosis in rats via modulating oxidative stress, inflammatory, fibrogenic and apoptotic markers. Biomed Pharmacother. (2018) 97:1356-65. doi: 10.1016/j.biopha.2017.11.028

23. Lee JS, Ryu JH, Elicker BM, Lydell CP, Jones KD, Wolters PJ, et al. Gastroesophageal reflux therapy is associated with longer survival in patients with idiopathic pulmonary fibrosis. Am J Respir Crit Care Med. (2011) 184:1390-4. doi: 10.1164/rccm.201101-0138OC

24. Pullamsetti SS, Savai R, Dumitrascu R, Dahal BK, Wilhelm J, Konigshoff $\mathrm{M}$, et al. The role of dimethylarginine dimethylaminohydrolase in idiopathic pulmonary fibrosis. Sci Transl Med. (2011) 3:87ra53. doi: 10.1126/scitranslmed.3001725

25. Ghebremariam YT, LePendu P, Lee JC, Erlanson DA, Slaviero A, Shah $\mathrm{NH}$, et al. Unexpected effect of proton pump inhibitors: elevation of the cardiovascular risk factor asymmetric dimethylarginine. Circulation. (2013) 128:845-53. doi: 10.1161/CIRCULATIONAHA.113.003602

26. Hammond CL, Roztocil E, Phipps RP, Feldon SE, Woeller CF. Proton pump inhibitors attenuate myofibroblast formation associated with thyroid eye disease through the aryl hydrocarbon receptor. PLoS ONE. (2019) 14:e0222779. doi: 10.1371/journal.pone.0222779

27. Gartland A, Rumney RM, Dillon JP, Gallagher JA. Isolation and culture of human osteoblasts. Methods Mol Biol. (2012) 806:337-55. doi: 10.1007/978-1-61779-367-7_22

Disclaimer: This content is solely the responsibility of the author and does not necessarily represent the official views of the sponsors.

Conflict of Interest: YG is an inventor on patents, owned by Stanford University and Baylor College of Medicine, that protect the use of agents, including proton pump inhibitors (PPIs), for therapeutic use of new indications.

Copyright $(2020$ Ghebre. This is an open-access article distributed under the terms of the Creative Commons Attribution License (CC BY). The use, distribution or reproduction in other forums is permitted, provided the original author(s) and the copyright owner(s) are credited and that the original publication in this journal is cited, in accordance with accepted academic practice. No use, distribution or reproduction is permitted which does not comply with these terms. 